\title{
Comprehensive analysis of multiprotein bridging factor 1 family genes and SIMBF1C negatively regulate the resistance to Botrytis cinerea in tomato
}

\author{
Xu Zhang, Zhixuan Xu, Lichen Chen and Zhonghai Ren*
}

\begin{abstract}
Background: Multiprotein bridging factor $1 \mathrm{~s}$ (MBF1s) are members of the transcriptional co-activator family that have involved in plant growth, development and stress responses. However, little is known about the Solanum lycopersicum MBF1 (SIMBF1) gene family.

Results: In total, five SIMBF1 genes were identified based on the tomato reference genome, and these genes were mapped to five chromosomes. All of the SIMBF1 proteins were highly conserved, with a typical MBF1 domain and helix-turn-helix_3 domain. In addition, the promoter regions of the SIMBF1 genes have various stress and hormone responsive cis-regulatory elements. Encouragingly, the SIMBF1 genes were expressed with different expression profiles in different tissues and responded to various stress and hormone treatments. The biological function of SIMBF1C was further identified through its overexpression in tomato, and the transgenic tomato lines showed increased susceptibility to Botrytis cinerea (B. cinerea). Additionally, the expression patterns of salicylic acid (SA)-, jasmonic acid (JA)- and ethylene (ET)- mediated defense related genes were altered in the transgenic plants.

Conclusions: Our comprehensive analysis provides valuable information for clarifying the evolutionary relationship of the SIMBF1 members and their expression patterns in different tissues and under different stresses. The overexpression of SIMBFIC decreased the resistance of tomato to $B$. cinerea through enhancing the gene expression of the SA-mediated signaling pathway and depressing JA/ET-mediated signaling pathways. These results will facilitate future functional studies of the transcriptional co-activator family.
\end{abstract}

Keywords: Tomato, MBF1, Expression pattern, SIMBF1C, Botrytis cinerea

\section{Background}

Transcriptional regulation is a key step in the expression of genomic information during complex biological processes in all organisms. Transcriptional co-activators are important components of gene expression that function by interacting with transcription factors and/or other regulatory elements and the basal transcription machinery [1]. Multiprotein bridging factor 1 (MBF1) proteins

\footnotetext{
* Correspondence: zhren@sdau.edu.cn

State Key Laboratory of Crop Biology, Shandong Collaborative Innovation Center of Fruit \& Vegetable Quality and Efficient Production, Key Laboratory of Biology and Genetic Improvement of Horticultural Crops in Huang-Huai Region, Ministry of Agriculture, College of Horticultural Science and Engineering, Shandong Agricultural University, Tai'an 271018, Shandong,
} China

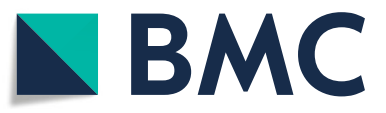

(c) The Author(s). 2019 Open Access This article is distributed under the terms of the Creative Commons Attribution 4.0 International License (http://creativecommons.org/licenses/by/4.0/), which permits unrestricted use, distribution, and reproduction in any medium, provided you give appropriate credit to the original author(s) and the source, provide a link to the Creative Commons license, and indicate if changes were made. The Creative Commons Public Domain Dedication waiver (http://creativecommons.org/publicdomain/zero/1.0/) applies to the data made available in this article, unless otherwise stated. are members of the transcriptional co-activator family and are highly conserved in eukaryotic organisms. MBF1 mediates the transcriptional activation of downstream genes by bridging regulatory transcription factors and TATA-box-Binding Protein [2]. MBF1 proteins are composed of a N-terminal domain, a conservative helixturn-helix (HTH) domain and a short C-terminus [3]. The HTH domain is critical to maintain the functional activity of MBF1 [4].

Several MBF1 genes have been identified in plants and have been shown to participate in plant growth, development and stress response. For example, Arabidopsis thaliana has three $M B F 1$ genes, and the expression levels of these genes have been found to be induced by 
various types of abiotic and biotic stress [2, 5, 6]. Arabidopsis plants that overexpress Arabidopsis thaliana MBF1a (AtMBF1a) show higher tolerance to salt stress and infection of pathogens, and they display a phenotype of hypersensitivity to Glucose [7]. The overexpression of AtMBF1c could enhance the tolerance to high temperature in Arabidopsis [8, 9]. The ectopic expression of Vitis labrusca x $V$. vinifera MBF1 in Arabidopsis increased drought tolerance [10], and the ectopic expression the Triticum aestivum MBF1c improved thermotolerance in rice [11]. However, not all of the $M B F 1$ genes are positive regulators that can enhance tolerance to environmental stress in plants. For example, Capsicum annuum MBF1 -overexpressing Arabidopsis lines have larger leaves but display sensitivity to cold and salt stress [12].

The tomato is one of the most widely cultivated vegetable crops in the world and a key model plant for the study of gene function [13]. However, the yield of tomato is seriously constrained by phytopathogens such as Botrytis cinerea (B. cinerea). Although the function of SlER24, a MBF1 family member, has been characterized and demonstrated to play an important role in tomato seed germination [14], the function of these genes except SIER24 were few reported. In our study, in order to explore the gene number of the $S I M B F 1$ family in tomato, a systematic analysis was performed in tomato with the tomato genome database. A total of five SIMBF1 proteins were identified. The phylogenetic results and motif analysis showed that the SIMBF1 family was highly conserved. In addition, an analysis of promoter response elements and the expression profiling of the SlMBF1s revealed marked responses to various hormones and stresses. Moreover, we obtained transgenic lines in the tomato. The overexpression of $S I M B F 1 c$ reduced the resistance of tomato to $B$. cinerea, suggesting SlMBF1c functions as a negative regulator in the tomato resistance to $B$. cinerea. Overall, the present study laid the foundation for the further study of $M B F 1$ genes, and their potentially use for trait improvement in the tomato.

\section{Results}

Identification and chromosomal location of SIMBF1 genes in the tomato

To identify the putative $M B F 1$ genes in the tomato genome, we used the three Arabidopsis MBF1 protein sequences and the conserved MBF1 and HTH_3 domains as queries to search the tomato genome database using the BlastP program (Additional file 3: Table S3). A total of five putative SIMBF1 proteins were obtained with default parameters. Then, the existence of the conserved MBF1 and HTH_3 domains was confirmed by SMART and CD-Search. As described by Sanchez-Ballesta et al.
[15], the four SlMBF1 genes were named SlMBF1a to $c$ and SIER24, and the newly identified SIMBF1 gene was named SlMBF1d.

The molecular property analysis revealed that these SIMBF1 proteins display similar lengths (139 amino acid for SIMBF1a, SIMBF1b, SIMBF1d, and 146 amino acid for SlER24). The predicted molecular weights of the five SIMBF1 proteins ranged from 15.272 (SIMBF1b) to 16.033 (SIER24) Dalton (Da). The predicted pI values ranged from 9.95 (SIMBF1a and SIMBF1d) to 10.11 (SlER24). The gene IDs and genomic positions were summarized for these SIMBF1 proteins (Additional file 1: Table S1). By analyzing the genomic location information obtained from tomato genome database, these five SlMBF1 genes were mapped on tomato chromosome 1, 7, 9, 10, 12, respectively (Fig. 1.a).

\section{Phylogenetic analysis, gene structure and conserved motifs of the SIMBF1 genes}

The full sequences of the five SIMBF1, three AtMBF1, and two OsMBF1 proteins were used to perform protein sequence alignment and phylogenetic analysis (Fig. 1b). These MBF1 proteins were defined as members of the other corresponding plants MBF1 subgroups [16]. Among these two subgroups, subgroup I is composed of four SIMBF1, one OsMBF1 and two AtMBF1 proteins, and subgroup II composed of one OsMBF1, one SIMBF1 and one AtMBF1 proteins. Due to evolutionary differences between these three species, subgroup I could be further divided into two groups, subgroup I-A and subgroup I-B. Among them, subgroup I-A included only four tomato SIMBF1 proteins and subgroup I-B included one OsMBF1 and two AtMBF1 proteins.

The gene structure analysis of the $M B F 1$ family genes from the tomato, Arabidopsis and rice were conducted and the results are consistent with the phylogenetic tree analysis. As shown in Fig. 1b, the number of exons in the SlMBF1, AtMBF1 and OsMBF1 genes ranges from one to five exons. We found that the two subgroups, subgroup II and subgroups I-B, have similar intron-exon structures (Fig. 1b). The three members, OsMBF1c, SlER24 and AtMBF1c, in subgroup II contain one exon, and the members, OsMBF1a, AtMBF1a and AtMBF1b, in subgroups I-B four exons. However, in subgroups I-A, SlMBF1b and SlMBF1c contain four exons, while SlMBF1a five exons and SlMBF1d one exon (Fig. 1b).

The motif analysis of the MBF1 proteins was conducted and four distinct motifs were identified (Fig. 1b; c and Additional file 2: Table S2). Motif 2 and 3, which are MBF1 domains, and motif 1, which is an HTH_3 domain, were identified in all MBF1 proteins. Interestingly, motif 4 was only identified in the SIMBF1a and OsMBF1c proteins. Therefore, the similar motif distribution of the MBF1 proteins in these three model plants 


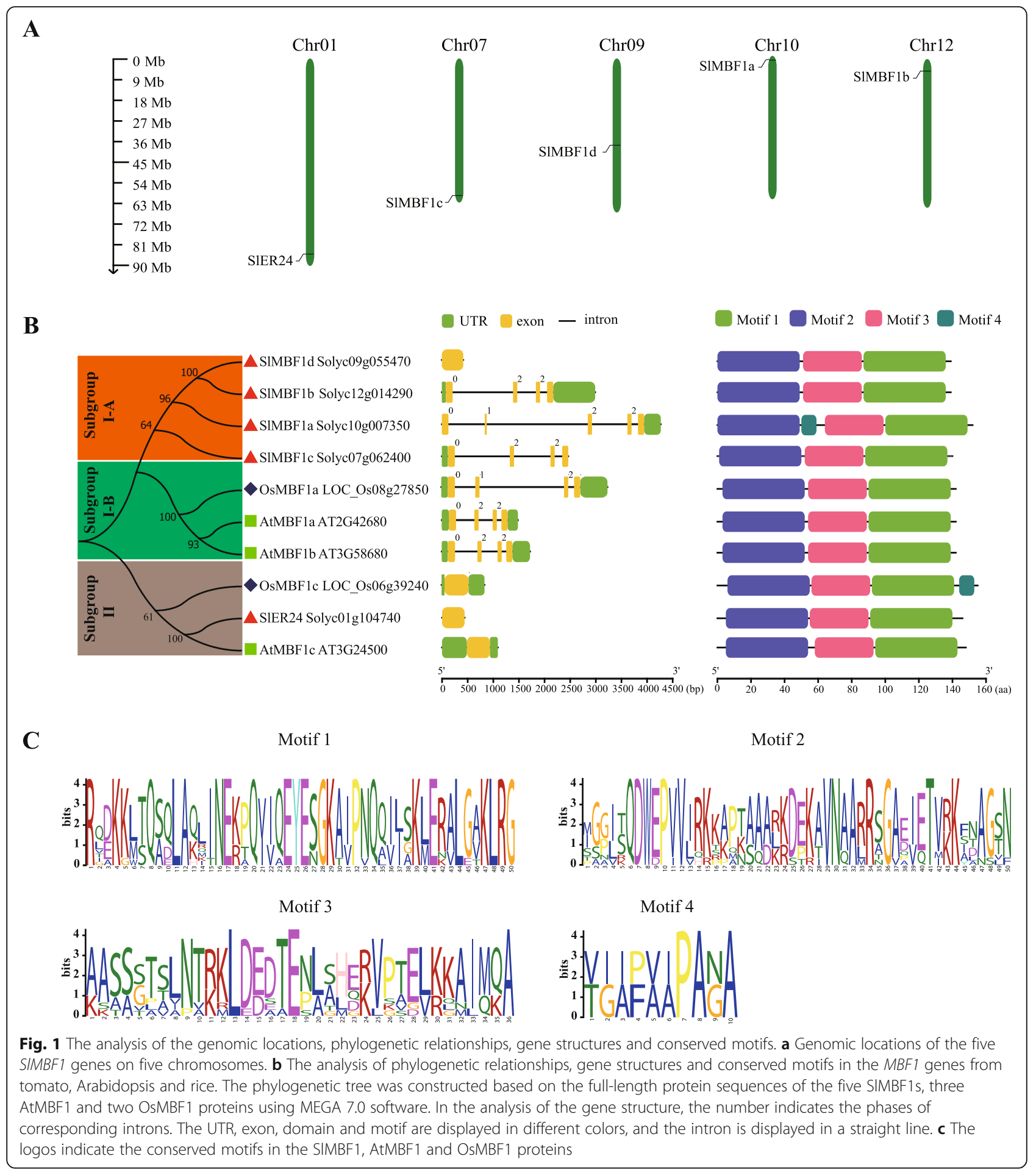

may promote to the prediction of the functions of MBF1s.

\section{Potential cis-elements in the promoters of SIMBF1 genes} Previous studies have shown that many $M B F 1$ genes play regulatory roles in developmental processes and tolerance to environmental stresses in plants. To predict the putative functions of the SIMBF1 genes, the 2.0-kb promoter regions of the SIMBF1 genes were isolated for the analysis of the potential cis-elements using the PlantCARE database (Fig. 2), and many elements related to stress responsiveness and plant hormones were predicted. As shown in Fig. 2, the promoters of SlMBF1 genes contain many stress elements: drought response 


Fig. 2 The promoter analysis of the SIMBF1 members in the tomato. The potential cis-regulatory elements in the promoter regions 2.0-kb
upstream of the SIMBF1s genes, particularly the elements related to stress responsiveness and plant hormones, are shown. Different shapes and
colors indicate whether the motif exists in the plus or minus strand of the cis-acting elements
TC-rich (defense and stress response element)

element, low temperature response element and defense and stress response element. Moreover, hormone responsive elements including abscisic acid (ABA) response element, gibberellin (GA) response element, jasmonate acid (MeJA) response element, salicylic acid (SA) response element and auxin response element were also discovered in the SIMBF1 promoters. These results suggest that the five $S I M B F 1$ genes may play important roles in the response to several hormones and various stresses.

\section{Expression pattern of the SIMBF1 genes in different tissues}

To understand the potential function of the tomato SlMBF1 genes, the expression pattern of these five SlMBF1 genes were examined using qRT-PCR in different tomato organs, including the root, stem, leaf, flower and ripe fruit. As shown in Fig. 3, all of the $S I M B F 1$ genes were detected in these five tissues. The expression of SIMBF1a, SlMBF1b and SIMBF1c were at relatively high levels in most tissues, but SlMBF1d was expressed at relatively lower levels in all tissues. SIER24 was expressed at relatively lower levels in root, stem and leaves but at relatively high levels in fruit and flower.

Expression pattern of SIMBF1 genes under different stress and different plant hormone conditions

To explore whether these five SIMBF1 genes respond to biotic and abiotic stresses in tomato, we examined the expression pattern of the SIMBF1 genes under different stress conditions, including salt, drought, low temperature, B. cinerea and wounding using qRT-PCR (Fig. 4). As
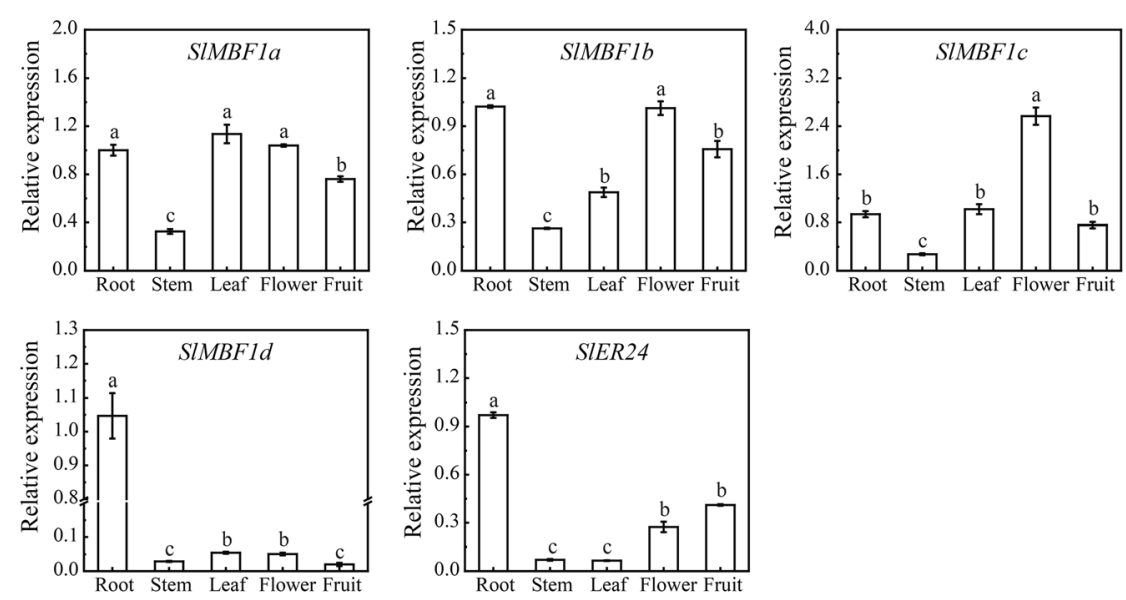

Fig. 3 Relative expression analysis of the SIMBF1 genes in different tissues. The expression levels of SIMBF1s in the root, stem, young leaf, flower, and ripe fruit using qRT-PCR analysis. Different letters indicate significant differences $(P<0.05)$ 


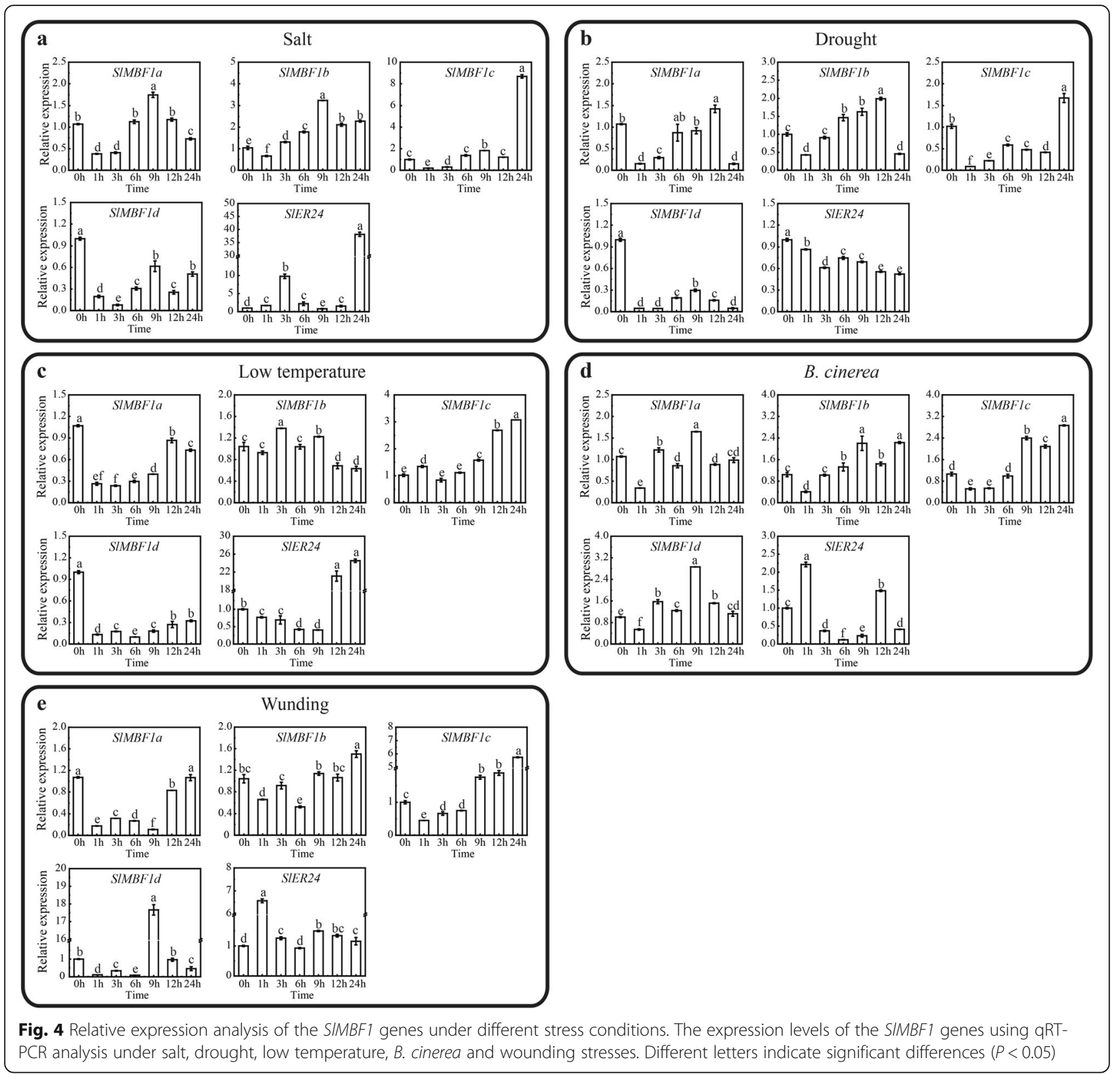

expected, most of the $S I M B F 1$ genes responded to different stress treatments. For example, SlMBF1c was induced during the late stage of all stress treatments (Fig. 4). The expression level of SIER24 was upregulated during the late stage of the salt and low temperature conditions (Fig. 4a, c). The expression level of SIMBF1a was initially downregulated then upregulated and then downregulated at the late stage under drought and $B$. cinerea conditions (Fig. $4 \mathrm{~b}, \mathrm{~d})$. Moreover, $S I M B F 1 b$ displayed the same expression trend with SIMBF1a under drought conditions (Fig. 4c).

To further study how these five SIMBF1 genes respond to plant hormones in the tomato, we also examined the expression pattern of the SlMBF1 genes under different hormone treatments, including 1-amino cyclopropane-1carboxylic acid (ACC), salicylic acid (SA), methyl jasmonate acid (MeJA), abscisic acid (ABA), and brassinosteroids (BR) using qRT-PCR (Fig. 5). As shown in Fig. 5, most of the SIMBF1 genes responded to different hormones. For example, the expression level of SIMBF1a and SIMBF1c was initially upregulated then downregulated at late stage under ACC and MeJA conditions. In contrast, the expression level of SlMBF1a was initially induced then repressed at the late stage under ACC and MeJA conditions. Some of the SlMBF1 genes were also induced under the SA, $\mathrm{ABA}$ and BR conditions (Fig. 5c, d, e). 


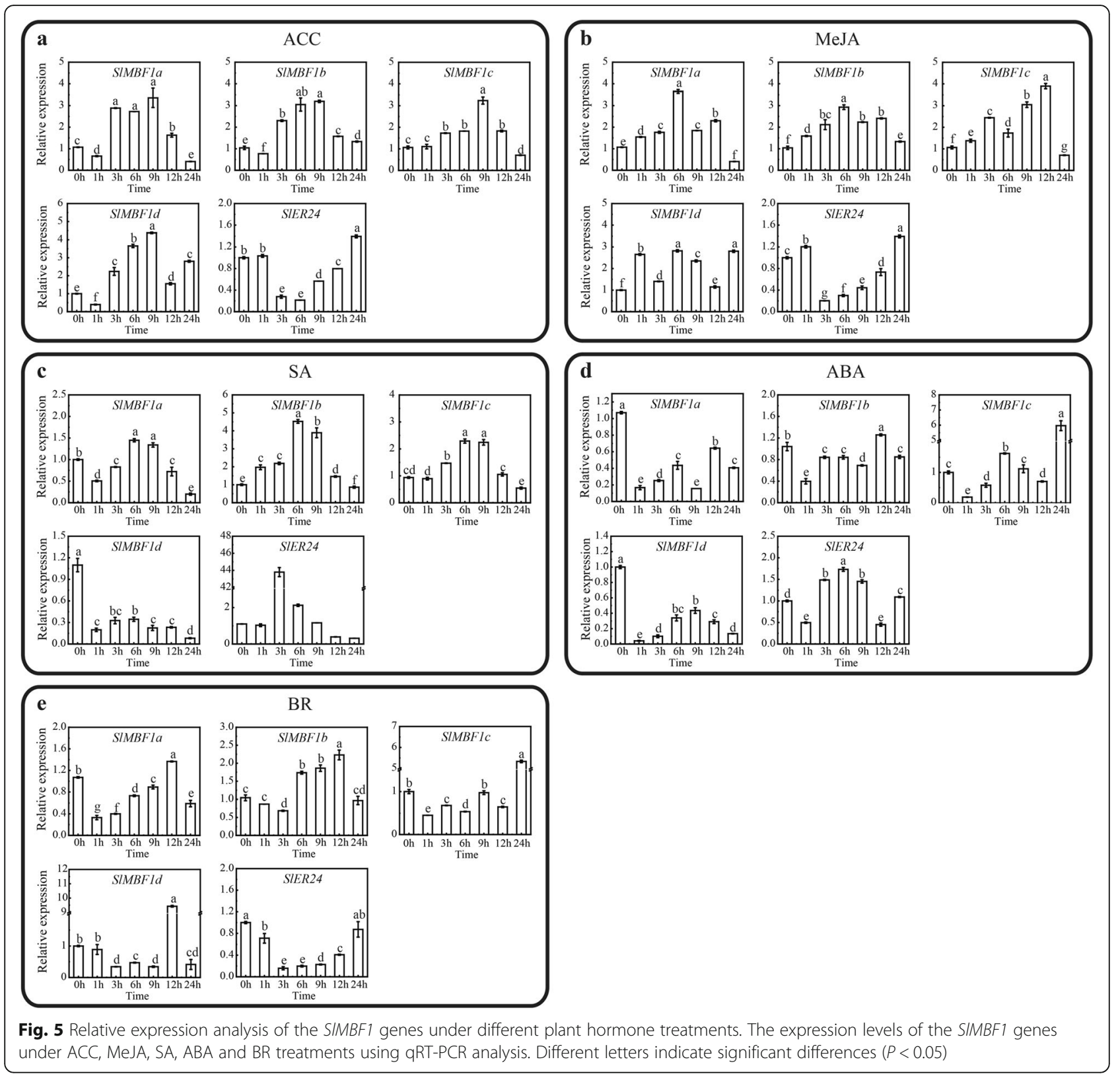

The susceptibility of SIMBF1C overexpressing lines to $B$. cinerea

To investigate the function of SlMBF1c in the defense response to $B$. cinerea, we generated 35S::SlMBF1c transgenic tomato plants (OE) by the Agrobacteriummediated method. Using kanamycin as selection marker and genomic PCR detection, two independent and homozygous $\mathrm{T} 3$ transgenic lines were selected for further assays. These two OE lines display significantly higher expression levels of SlMBFIc than the WT plants (Fig. 6). Then, we examined the response of the leaves from 5-week-old OE and WT seedlings to $B$. cinerea infection in Petri dishes, using the method previously described by Du et al., 2017 [17]. As shown in Fig. $7 \mathrm{a}$ and $\mathrm{b}$, after infection with $B$. cinerea, the OE leaves showed significantly larger necrotic lesions compared with WT. Moreover, we also conducted the whole plant inoculation experiments. Similarly, the OE plants displayed a sensitive phenotype, compared with WT, after infection with $B$. cinerea (Fig. $7 \mathrm{c}, \mathrm{d}$ and e). In addition, the expression level of B. cinerea Actin was significantly increased in OE plants compared with WT (Fig. 7 f). Taken all together, these results demonstrated that tomato $S l M B F 1 c$ is a negative regulator in the response to $B$. cinerea infection. 


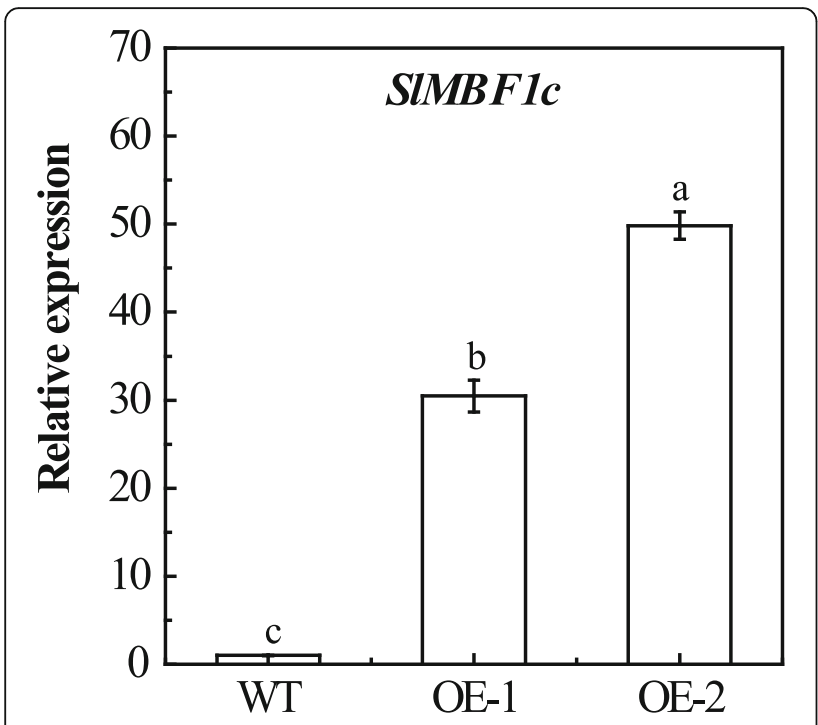

Fig. 6 Characterization of the SIMBFIC transgenic tomato plants. The leaves of T3 SIMBF1C-overexpressing and WT tomato plants were used for the qRT-PCR analysis. The actin gene was used as an internal control to normalize all data. Different letters indicate significant differences $(P<0.05)$

\section{SIMBF1c regulates the expression of defense-related genes}

To explore the signaling pathways, we analyzed and compared the changes in the relative expression of SA signaling-related genes Nonexpressed PathogenesisRelated 1 (SINPR1) and Pathogenesis-Related genes (SlPR1a, SlPR1b and SIPR2b), JA signaling-related genes Coronatin Insensitive 1 (SlCOI1), Myelocytomatosis Oncogene 2 (SIMYC2), Proteinase Inhibitor I (SIPI I) and Leucine Aminopeptidase A1 (SlLapA1), and ET signaling-related genes Ethylene Response Factor 1 (SlERF1), Ethylene Receptor (SINR), ACC Synthase 6 (SlACS6) and Allene Oxide Synthase 2 (SlAOS2) before and after infection with $B$. cinerea using qRT-PCR. As shown in Fig. 8, before infection, the transcript levels of SINPR1, SlPR1a, SlPR2b, SlCOI1, SIPI I and SlACS6 display no significantly difference between the two OE lines and WT. However, the transcript levels of SlPR1b, SIERF1, SINR, SlAOS2 were increased slightly and the transcript levels of SlLapA1 were decreased slightly in the OE lines. After infection with $B$. cinerea, the transcriptional levels of SA signaling-related genes (SINPR1, SIPR $1 a, S I P R 1 b$ and SlPR $2 b$ ) were elevated significantly in the two OE lines compared with WT (Fig. 8a). However, after infection with $B$. cinerea, the expression levels of the JA signaling-related gene (SlCOI1, SIMYC2, SIPII and SlLapA1) and the ET signaling-related genes (SlERF1, SlNR, SlACS6 and SlAOS2) were significantly decreased in the two OE lines compared with WT (Fig. $8 \mathrm{~b}$ and $\mathrm{c}$ ). These results indicated that the overexpression of SIMBF1c in the tomato could repress the JA/ET- mediated signaling pathways upon infection with $B$. cinerea.

\section{Discussion}

With the genomes of more species completely sequenced, many regulatory gene families such as the MYB [18], bHLH [19] and WRKY [20] transcription factor families, have been identified. In addition to these transcription factor families, there are also transcriptional co-activator families such as MBF1s. Studies of $M B F 1$ genes have mainly focused on the regulation of plant growth, development and stress responses in Arabidopsis [2, 7, 8]. Although in the year 2007, SanchezBallesta et al. identified four $M B F 1$ genes in the tomato and analyzed their structures, tissue-specific expression and response to ethylene treatment during fruit development [15], the tomato genome sequence completed in 2012 provides more information for the identification of this gene family [13]. Here, five tomato $M B F 1$ genes were identified and confirmed based on the completed tomato genome (Fig. 1a). Meanwhile, the more precise and comprehensive bioinformatics analysis (including the chromosomal location, phylogenetic analysis, gene structure, conserved motifs and cis-elements in the promoters) were performed. Notably, we found five exons in the gene structure of SIMBF1a, but Sanchez-Ballesta et al. only found four exons. Comprehensive expression levels of these genes in different tissues, responses to different stresses (salt, drought, low temperature, $B$. cinerea and wounding) and different plant hormone conditions (ACC, MeJA, SA, ABA and BR) were also detected (Figs. 4 and 5). More importantly, we identified the biological function of $S I M B F 1 c$ which negatively regulate the tomato resistance to B. cinerea (Figs. 6,7 and 8).

In this study, five $M B F 1$ genes were distributed on five chromosomes of tomato, respectively (Fig. 1a). Compared with three MBF1s in Arabidopsis [2] and two $M B F 1 s$ in rice, the number of $M B F 1 s$ was greater in the tomato, which means an expansion of $M B F 1 s$ in tomato. A phylogenetic analysis divided these $10 \mathrm{MBF} 1$ proteins into two main branches (Fig. 1b), the same as in previous description [16]. One branch contained subgroup I$A$ and $B$, and the other contained subgroup II (Fig. 1b). This result revealed that there are two different evolutionary directions for these MBF1 proteins in tomato, Arabidopsis and rice. Importantly, subgroup I-A only includes four MBF1 proteins but did not include any Arabidopsis or rice MBF1 proteins (Fig. 1b), which means that this subgroup was lost in Arabidopsis and rice and was acquired in tomato after divergence from the last common ancestor. Moreover, the gene structure analysis showed similar intron-exon structures in subgroup I-B and subgroup II but not in subgroup I-A (Fig. 1B), suggesting that the evolutionary dynamics of intron 


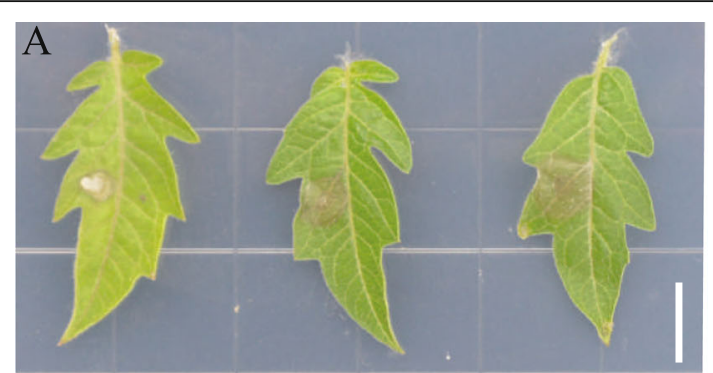

B
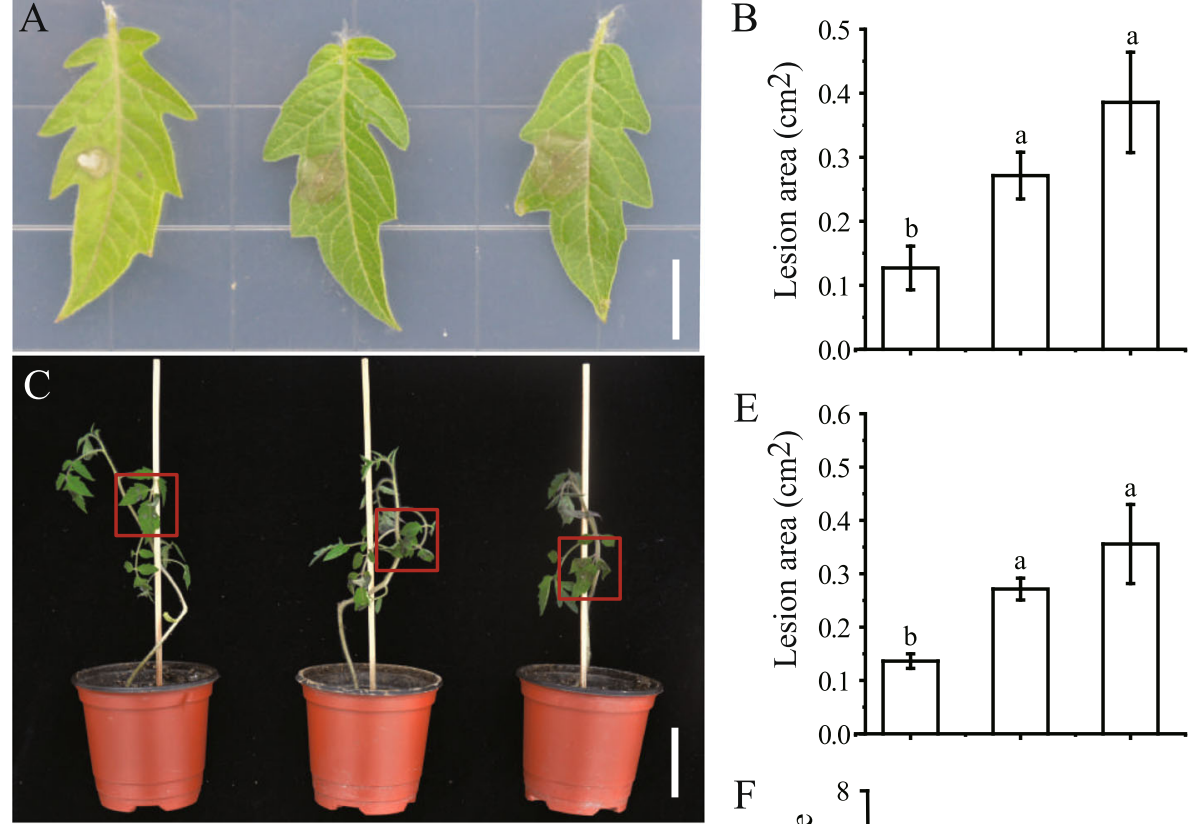

$\mathrm{E}$
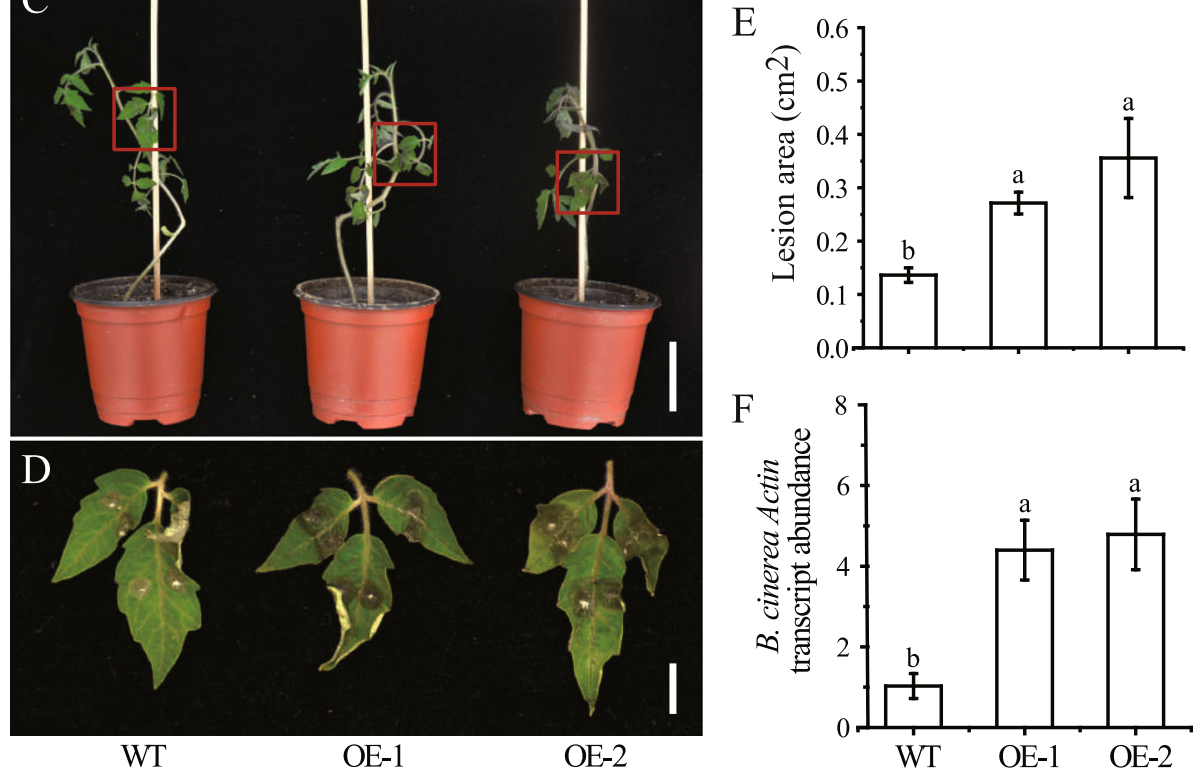

Fig. 7 Overexpression of SIMBFIC resulted in decreased resistance to $B$. cinerea. a The response of wild-type and SIMBFIC-OE plant leaves to $B$. cinerea infection at $2 \mathrm{dpi}$ in Petri dishes (Scale bars, $1 \mathrm{~cm}$ ). $\mathbf{b}$ The quantification of lesion areas on the leaves shown in (a). $\mathbf{c}$ and $(\mathbf{d})$ The response of whole plants of wild-type and SIMBFIC-OE to B. cinerea infection at $2 \mathrm{dpi}$ (Scale bars, $5 \mathrm{~cm}$ and $1 \mathrm{~cm}$, respectively). e The quantification of lesion areas on the leaves shown in (c). $\mathbf{f}$ Relative transcript abundance of the $B$. cinerea Actin in the infected leaves from the whole plant inoculation experiments at $2 \mathrm{dpi}$. Detached leaves from 5-week-old tomato plants were spotted with $5 \mu \mathrm{l}$ of spore suspension $\left(10^{6} \mathrm{spores} / \mathrm{ml}\right)$. The results in (b), e and (f) are presented as the mean values $\pm S D ; n=$ six leaves from different plants. Different letters indicate significant differences between treatments $(P<0.05)$

insertion and loss occurred in subgroup I-A of the tomato $M B F 1$ genes. Previous studies have shown that the yeast $m b f 1$ mutant was fully/partially rescued by the MBF1 genes from human, silkworm and Arabidopsis [2, 21], which revealed that the functions of the $M B F 1$ genes are highly conserved. In this study, the motif analysis showed that these MBF1 proteins share similar pattern of motif composition and that all of them have MBF1 and HTH_3 domains (Fig. 1b), which means that the function of MBF1 proteins among tomato, Arabidopsis and rice might be similar and conserved.

The expression pattern analysis in different tissues, stresses and plant hormones is usually used to predict the potential functions of genes in plant growth, development and the responses to stresses. Through the expression pattern analysis, we found that all of the SlMBF1s genes were expressed in the five tissues, and most of them had much higher expression in the flower and leaf (Fig. 3). In addition, GA response element was found in the promoter regions of these five SIMBF1 genes and IAA response element also in the promoter regions of SlMBF1a, SlMBF1b and SIER24 (Fig. 2). These results indicated that SIMBF1s might be involved in plant growth and development. Besides the roles in plant growth and development [14], MBF1 genes also participate in the responses to abiotic and biotic stresses, such as salt, drought, temperature and pathogens [512]. Indeed, several stress-related elements (drought, low temperature, ABA, defense and stress, JA and SA response elements) were found in the promoter regions of these SlMBF1s (Fig. 2). In addition, SlMBF1 genes were induced by abiotic and biotic stresses (e.g. salt, drought, cold and B. cinerea) and by stress-related hormones (e.g. ABA, SA, JA and ACC) (Figs. 4 and 5). These results 


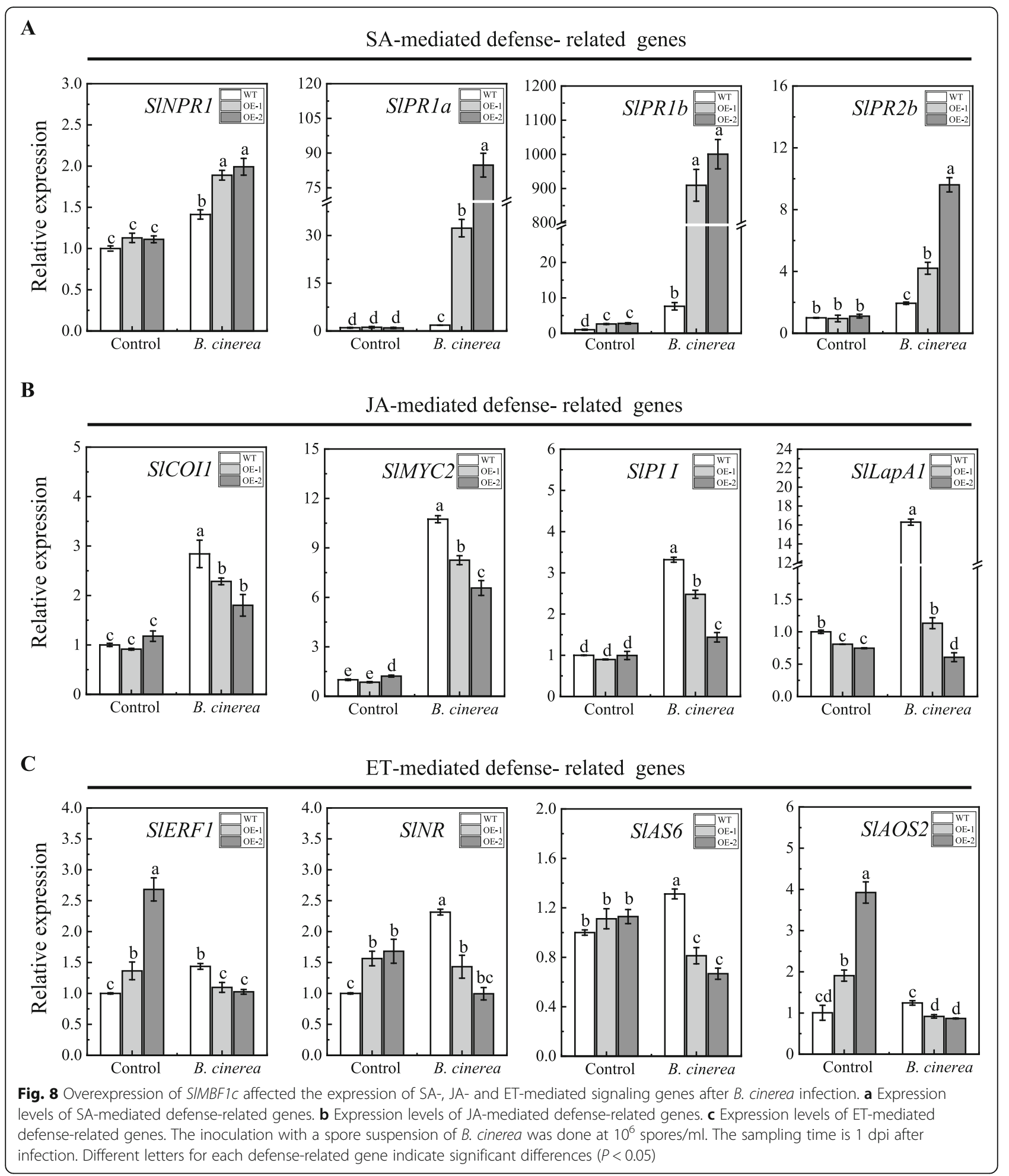

indicated that these SlMBF1 genes were involved in the responses to stresses with the functions similar to the $M B F 1 s$ from other species [7-12].

The tomato is an important economic and vegetable crop. However, $B$. cinerea seriously limits the yield of tomato [22]. In this study, the expression of SlMBF1c was significantly induced by $B$. cinerea, wounding and defense-signaling related hormones (Figs. 4 and 5). Additionally, several defense related elements were also found in the promoter of SlMBF1c (Fig. 2). Moreover, 
overexpressing the AtMBF1a gene in Arabidopsis confers increased resistance under the infection by $B$. cinerea [7]. In order to clarify the function of SlMBF1c in the defense response, tomato plants overexpressing SlMBF1c were generated. To our surprise, the transgenic lines displayed a sensitive phenotype, as compared with WT, under infection with $B$. cinerea (Fig. 6). The finding that $S I M B F 1 c$ regulates the resistance to $B$. cinerea is distinct from the function of its Arabidopsis homolog. This phenomenon might be due to the evolutionary differences between SIMBF1c and AtMBF1a, because SlMBF1c belonged to subgroup I-A, but AtMBF1a to subgroup I-B in the phylogenetic tree (Fig. 1b).

Previous studies showed that $B$. cinerea can activate the SA signaling pathway to promote its pathogenicity in plants [23, 24]. Meanwhile, plants can activate the JA/ ET-mediated defense responses against $B$. cinerea infection $[23,25,26]$. However, the SA signaling pathway can antagonize the JA/ET signaling pathways in plants under $B$. cinerea infection $[23,25]$. In our study, under control condition, only SIPR $1 b$ in the SA signaling pathway showed slightly higher expression in the OE lines compared with WT, but under $B$. cinerea infection, all of SINPR1, SIPR1a, SIPR $1 b$ and SlPR2b were significantly up-regulated in OE lines (Fig. 8a). Moreover, the overexpression of SIMBF1c further promote the expression levels of the SA signaling pathway genes, especially SlPR1a and $b$ (Fig. 8a). On the contrary, under control condition, only SlLapA1 in the JA signaling pathway showed slightly lower expression in the OE lines compared with WT; but under $B$. cinerea infection, all of SlCOI1, SlMYC2, SlPI I and SlLapA1 were significantly down-regulated in OE lines (Fig. 8b). These results suggested that the JAmediated defense responses in infected OE lines was seriously suppressed by the highly activated the SA signaling pathway under B. cinerea infection (Fig. 8b) [26]. In addition, although under control condition, the ET-mediated defense genes (SIERF1, SINR, SlACS6 and SlAOS2) showed higher expression in the $\mathrm{OE}$ lines compared with WT (Fig. 8c), all of these genes were significantly down-regulated in the infected $\mathrm{OE}$ lines, indicating that the ET signaling pathway in the infected $\mathrm{OE}$ lines was also greatly suppressed by the highly activated SA signaling (Fig. 8c). Taken together, these results clarified that the SlMBF1c-overexpressing tomato plants displayed a sensitive phenotype due to the strongly activated SA pathway which antagonized the JA/ET-mediated defense responses under $B$. cinerea infection.

NPR1 is not only a master regulator of SA signaling, but also a key regulator in the antagonism between SA and JA through suppressing the JA signaling gene PI I [23, 27]. Indeed, the expression level of SINPR1 was increased and SIPI I repressed in the infected OE lines (Fig. 8a-b), suggesting that $S I M B F 1 c$ could activate SINPR1 to repress SIPI I in infected OE lines. This result is consistent with the previous study [26]. However, in our study, the expression levels of SIPR1a and SIPR $1 b$ were more dramatically increased than SINPR1 in the infected OE lines, and SlLapA1 more dramatically decreased than SIPI I (Fig. 8a-b). Therefore, it will be interesting to clarify that how SIPR1a and SIPR1b are induced and whether SIPR1a and/or SIPR1b are the new key regulators to suppress SlLapA1 in the antagonism between SA and JA signaling pathways when $S I M B F 1 c$ is being overexpressed in the tomato under $B$. cinerea stress condition in the future studies.

\section{Conclusion}

In this study, five SlMBF1 genes including SlMBF1d newly-found were identified and confirmed in the tomato genome. The analysis of phylogenetic tree, gene structures and protein motifs revealed that MBF1 proteins are conserved among tomato, Arabidopsis and rice and expanded in the tomato. The cis-elements in the promotors, tissue specific expression pattern and responses to stresses and hormones suggested that the SlMBF1s might participate in plant growth and development and stress responses in the tomato. Finally, transgenic experiments showed that SlMBF1c negatively regulate the tomato resistance to $B$. cinerea through enhancing SA-signaling genes and repressing the genes in the JA/ET-mediated pathways.

\section{Methods}

\section{Identification of MBF1 genes in the tomato}

To identify the SlMBF1 gene family members from the entire tomato genome, three AtMBF1 proteins were used as query sequences for Blastp searches with an evalue of $10^{-10}$ against the predicted tomato proteins. In addition, the Hidden Markov Model (HMM) profile of MBF1 (PF08523.9) and HTH_3 (PF01381.21) from the Pfam database (http://pfam.janelia.org) were also applied as queries to search the $M B F 1$ genes from the tomato genome database (http://solgenomics.net; ITAG Release 3.20). In order to identify the conserved domains, five candidate genes were further confirmed due to the presence of both the MBF1 (PF08523.9) and HTH_3 (PF01381.21) domains using the Pfam database and SMART database (http://smart.embl-heidelberg.de).

The MBF1 proteins in the representative model plants Arabidopsis and rice were downloaded from The Arabidopsis Information Resource database (https://www.arabidopsis.org) and the Rice Genome Annotation Project Database (http://rice.plantbiology.msu.edu/). 


\section{Phylogenetic analysis}

The multiple sequence alignment was constructed by Clustal W (version 1.81, a resident software, European Molecular Biology Laboratory, Heidelberg, Germany.) with default parameters [28]. Full sequences of five SlMBF1, thress AtMBF1 and two OsMBF1 proteins were used to construct the phylogenetic tree using MEGA v7.0 [29]. The Neighbor-Joining method was used with the following parameters: Poisson correction, pairwise deletion, and bootstrap (1000 replicates; random seed) [30].

Analysis of physical properties, chromosomal localization, gene structure, conserved motif recognition and response elements in promoter regions

Physical properties such as theoretical protein isoelectric point (pI) and molecular weight of the SIMBF1 proteins were calculated using the ExPASy server's Compute pI/ Mw tool (http://web.expasy.org/compute_pi/) [31]. The information of the chromosomal locations and gene structures were downloaded from the tomato genome database. The conserved motifs were analyzed using MEME database (http://meme-suite.org/) [32]. Additionally, the response elements in the promoter regions were analyzed using the PlantCARE database (http://bioinformatics.psb.ugent.be/webtools/plantcare/html/) [33]. The chromosomal locations were visualized by Mapchart 2.30 software [34]. The gene structures, conserved motifs and response elements in the promoter regions were visualized by GSDS Server 2.0 (http://gsds.cbi.pku.edu.cn/).

\section{Plant materials and growth conditions}

Tomato (Solanum lycopersicum L. cv 'SN1' [35]) seedlings were grown in a biotron at Shandong Agricultural University with a $16 \mathrm{~h}$ light $\left(28^{\circ} \mathrm{C}\right) / 8 \mathrm{~h}$ dark $\left(22^{\circ} \mathrm{C}\right)$ photoperiod $\left(18.5 \mu \mathrm{mol} \mathrm{m}^{-2} \mathrm{~s}^{-1}\right)$. Four-week-old tomato seedlings were used for all types of treatments.

\section{Different stresses and hormone treatments}

For the salt and drought stress treatment assays, tomato plants (4-week-old) were transferred into the $10 \mathrm{~L}$ tanks containing half-strength Hoagland nutrient solution and were maintained in this system for one week before supplementation with $\mathrm{NaCl}(150 \mathrm{mM})$ and Polyethylene glycol 8000 (20\%), as previously described [36]. The tomato plants were transferred to the incubator for cold treatment at $4{ }^{\circ} \mathrm{C}$. The seedling leaves were pressed with hemostatic forceps for the wounding treatment. The inoculation of the tomato plants with B. cinerea (B05.10) was performed as previously described [17, 23, 37], with minor modifications. The seedling leaves were spotted with a single $5-\mu \mathrm{l}$ droplet of $B$. cinerea spore suspension $\left(10^{6}\right.$ spores $\left./ \mathrm{ml}\right)$ for the pathogen treatment. For the hormone treatments, the seedling leaves were sprayed with ACC $(100 \mu \mathrm{M})$, SA $(2 \mathrm{mM})$, MeJA $(100 \mu \mathrm{M})$, ABA
$(100 \mu \mathrm{M})$ and $\mathrm{BR}(200 \mu \mathrm{M})$. The leaves from different tomato plants were collected for the qRT-PCR analysis.

\section{RNA isolation and quantitative real-time PCR analysis}

The total RNA from tomato leaves was extracted with TRIzol Reagent (Invitrogen, Carlsbad, CA, USA) according to the manufacturer's instructions. The first-strand cDNA was synthesized from one microgram of total RNA using a reverse transcriptase system (Thermo, Beijing, China), according to the manufacturer's instructions. The reactions were performed using the SYBR Mixture (Juheme) with an Applied Biosystems 7500 real-time PCR system (Applied Biosystems). The PCR assays were conducted with the following parameters: $95^{\circ} \mathrm{C}$ for $30 \mathrm{~s} ; 40$ cycles of $95^{\circ} \mathrm{C}$ for 30 s, $60^{\circ} \mathrm{C}$ for $15 \mathrm{~s}$, and $72^{\circ} \mathrm{C}$ for $15 \mathrm{~s}$. All of the primers that were used in the qRT-PCR analysis are listed in Additional file 4: Table S4, some of which came from the previous studies [24, 38-40]. The tomato Actin2 gene was used as the internal control. The results were calculated using the $2^{-\Delta \Delta C t}$ method [41]. All of the qRT-PCR assays were conducted in three biological replicates and each biological replicate had three technical replicates.

\section{Vector construction and plant transformation}

For the construction of the overexpressing $S I M B F 1 c$ vector, the entire $S I M B F 1 c$ coding sequence was amplified using the primers SIMBF1c-F: TATCACAAGACTGG GAGC and SIMBF1c-R: GTCGTACTACTAGAGGCA. Then, the amplified products were digested with $\mathrm{XbaI}$ and KpnI sites and inserted into the pBI121 vector under the control of the 35S promoter. The 35S: SlMBF1c construct was transferred into the Agrobacterium strain LBA4404 by electroporation, and the Agrobacteriummediated tomato transformation was performed following the protocols described by Fillatti et al. [42].

\section{Statistical analysis}

All of the error bars for expression levels, represent the standard deviation (SD) which came from three technical replicates, except that in the phenotypic analysis of $\mathrm{OE}$ lines which came from six biological replicates. The analysis of significance level was performed with the Student's $t$-test at $p<0.05$ using Excel 2010 (Microsoft Cooperation, Washington, NJ, USA).

\section{Supplementary information}

Supplementary information accompanies this paper at https://doi.org/10. 1186/s12870-019-2029-y.

Additional file 1: Table S1. Molecular properties of SIMBF1 gene family in tomato.

Additional file 2: Table S2. Ten conserved motifs sequences and the bit score means information content from all MBF1 proteins from tomato, Arabidopsis and rice. 
Additional file 3: Table S3. The conserved domains information of five tomato MBF1 protein.

Additional file 4: Table S4. Primers used for $q R T-P C R$.

\section{Abbreviations}

aa: amino acid; ABA: Abscisic acid; ACC: 1-Amino cyclopropane-1-carboxylic acid; AtMBF1: Arabidopsis thaliana Multiprotein bridging factor $1 ; B$. cinerea: Botrytis cinerea; BR: Brassinosteroids; Da: Dalton; JA: Jasmonic acid; MBF1: Multiprotein bridging factor 1; MEGA: Molecular Evolutionary Genetics Analysis; MeJA: Methyl jasmonate; NJ: Neighbor-Joining; OE: Overexpression; OsMBF1: Oryza sativa Multiprotein bridging factor 1; pl: protein isoelectric point; SA: Salicylic acid; SIMBF1: Solanum lycopersicum Multiprotein bridging factor 1

\section{Acknowledgements}

We thank Professor Jiuhai Zhao, College of Agronomy, Shandong Agricultural University, for providing B. cinerea (B05.10). We also thank Professor Qinghua Shi, College of Horticultural Science and Engineering, Shandong Agricultural University, for providing tomato (Solanum lycopersicum L. CV 'SN1') seeds.

\section{Authors' contributions}

ZR designed the experiments. XZ performed the most of experiments. LC contributed to the plasmid constructions. $Z X$ involved the inoculation assays of $B$. cinerea. $X Z$ analyzed the data and wrote the manuscript. ZR revised the manuscript. All authors have read and approved this manuscript.

\section{Funding}

This work was supported by fundings from the National Natural Science Foundation of China (31672170 and 31872950), the Natural Science Foundation of Shandong Province (JQ201309), the Shandong "Double Tops" Program (SYL2017YSTD06) and the 'Taishan Scholar' Foundation of the People's Government of Shandong Province (ts20130932). The funds played no role in study design, data analysis, and manuscript preparation.

\section{Availability of data and materials}

The data that support the results are included within the article and its additional file. Other relevant materials are available from the corresponding authors on reasonable request.

\section{Ethics approval and consent to participate}

Not applicable.

\section{Consent for publication}

Not applicable.

\section{Competing interests}

The authors declare that they have no competing interests.

Received: 10 June 2019 Accepted: 11 September 2019

Published online: 21 October 2019

\section{References}

1. Naar AM, Lemon BD, Tjian R. Transcriptional coactivator complexes. Annu Rev Biochem. 2001;70:475-501.

2. Tsuda K, Yamazaki K. Structure and expression analysis of three subtypes of Arabidopsis MBF1 genes. BBA-Biomembranes. 2004;1680(1):1-10.

3. Wang $Y$, Wei $X$, Huang J, Wei J. Modification and functional adaptation of the MBF1 gene family in the lichenized fungus Endocarpon pusillum under environmental stress. Sci Rep. 2017;7(1):16333.

4. Ozaki J, Takemaru KI, Ikegami T, Mishima M, Ueda H, Hirose S, Kabe Y, Handa H, Shirakawa M. Identification of the core domain and the secondary structure of the transcriptional coactivator MBF1. Genes Cells. 2010;4(7):415-24.

5. Arce DP, Godoy AV, Tsuda K, Yamazaki K, Valle EM, Iglesias MJ, Di Mauro MF, Casalongue CA. The analysis of an Arabidopsis triple knock-down mutant reveals functions for $M B F 1$ genes under oxidative stress conditions. J Plant Physiol. 2010;167(3):194-200.

6. Suzuki N, Sejima $H$, Tam R, Schlauch $K$, Mittler R. Identification of the MBF1 heat-response regulon of Arabidopsis thaliana. Plant J. 2011;66(5):844-51.
7. Kim MJ, Lim GH, Kim ES, Ko CB, Yang KY, Jeong JA, Lee MC, Kim CS. Abiotic and biotic stress tolerance in Arabidopsis overexpressing the Multiprotein bridging factor 1a (MBF1a) transcriptional coactivator gene. Biochem Bioph Res Co. 2007:354(2):440-6.

8. Suzuki N, Bajad S, Shuman J, Shulaev V, Mittler R. The transcriptional coactivator $M B F 1 C$ is a key regulator of thermotolerance in Arabidopsis thaliana. J Biol Chem. 2008;283(14):9269-75.

9. Suzuki N, Rizhsky L, Liang H, Shuman J, Shulaev V, Mittler R. Enhanced tolerance to environmental stress in transgenic plants expressing the transcriptional coactivator multiprotein bridging factor 1c. Plant Physiol. 2005;139(3):1313-22

10. Yan $\mathrm{Q}$, Hou H, Singer SD, Yan X, Guo R. The grape VVMBF1 gene improves drought stress tolerance in transgenic Arabidopsis thaliana. Plant Cell Tiss Org. 2014;118(3):571-82

11. Qin D, Wang F, Geng X, Zhang L, Yao Y, Ni Z, Peng H, Sun Q Overexpression of heat stress-responsive TaMBFIC, a wheat (Triticum aestivum L.) multiprotein bridging factor, confers heat tolerance in both yeast and rice. Plant Mol Biol. 2015;87(1-2):31-45.

12. Guo WL, Chen RG, Du XH, Zhang Z, Yin YX, Gong ZH, Wang GY. Reduced tolerance to abiotic stress in transgenic Arabidopsis overexpressing a Capsicum annuum multiprotein bridging factor 1. BMC Plant Biol. 2014;14(1):138.

13. Consortium TTG. The tomato genome sequence provides insights into fleshy fruit evolution. Nature. 2012:485(7400):635-41.

14. Hommel M, Khalil-Ahmad Q, Jaimes-Miranda F, Mila I, Pouzet C, Latché A, Pech $J C$, Bouzayen M, Regad F. Over-expression of a chimeric gene of the transcriptional co-activator MBF1 fused to the EAR repressor motif causes developmental alteration in Arabidopsis and tomato. Plant Sci. 2008;175(1):168-77.

15. Sanchez-Ballesta MT, Hommel M, Jaimes-Miranda F, Tournier B, Zegzouti $H$, Mila I, Latché A, Pech JC, Bouzayen M, Regad F. Characterization of tomato SI-MBF1 transcriptional coactivator gene family. Advances in Plant Ethylene Research: Proceedings of the 7th International Symposium on the Plant Hormone Ethylene. 2007:369-75.

16. Tsuda K, Tsuji T, Hirose S, Yamazaki K-i. Three Arabidopsis MBF1 homologs with distinct expression profiles play roles as transcriptional co-activators. Plant Cell Physiol. 2004;45(2):225-31.

17. Du M, Zhao J, Dtw T, Liu Y, Deng L, Yang T, Zhai Q, Wu F, Huang Z, Zhou M. MYC2 orchestrates a hierarchical transcriptional cascade that regulates jasmonatemediated plant immunity in tomato. Plant Cell. 2017;29(8):1883-906.

18. Zhao P, Li Q, Li J, Wang L, Ren Z. Genome-wide identification and characterization of R2R3MYB family in Solanum lycopersicum. Mol Gen Genomics. 2014;289(6):1183-207.

19. Mao K, Dong Q, Li C, Liu C, Ma F. Genome wide identification and characterization of apple bHLH transcription factors and expression analysis in response to drought and salt stress. Front Plant Sci. 2017;8:480.

20. Xie T, Chen C, Li C, Liu J, Liu C, He Y. Genome-wide investigation of WRKY gene family in pineapple: evolution and expression profiles during development and stress. BMC Genomics. 2018;19(1):490.

21. Takemaru Kl, Li FQ, Ueda H, Hirose S. Multiprotein bridging factor 1 (MBF1) is an evolutionarily conserved transcriptional coactivator that connects a regulatory factor and TATA element-binding protein. PNAS. 1997;94(14):7251-6.

22. Dean R, Van Kan JA, Pretorius ZA, Hammond-Kosack KE, Di Pietro A, Spanu PD, Rudd JJ, Dickman M, Kahmann R, Ellis J, et al. The top 10 fungal pathogens in molecular plant pathology. Mol Plant Pathol. 2012;13(4):414-30.

23. El Oirdi M, El Rahman TA, Rigano L, El Hadrami A, Rodriguez MC, Daayf F, Vojnov A, Bouarab K. Botrytis cinerea manipulates the antagonistic effects between immune pathways to promote disease development in tomato. Plant Cell. 2011;23(6):2405-21.

24. Veronese P, Nakagami H, Bluhm B, Abuqamar S, Chen X, Salmeron J, Dietrich RA, Hirt H, Mengiste T. The membrane-anchored BOTRYTISINDUCED KINASE1 plays distinct roles in Arabidopsis resistance to necrotrophic and biotrophic pathogens. Plant Cell. 2006;18(1):257-73.

25. Glazebrook J. Contrasting mechanisms of defense against biotrophic and necrotrophic pathogens. Annu Rev Phytopathol. 2005;43(1):205-27.

26. Grant MR, Jones JDG. Hormone (dis) harmony moulds plant health and disease. Science. 2009;324(5928):750-2.

27. Koornneef A, Pieterse CMJ. Cross talk in defense signaling. Plant Physiol. 2008;146(3):839.

28. Thompson JD, Higgins DG, Gibson TJ. CLUSTAL W: improving the sensitivity of progressive multiple sequence alignment through sequence weighting, position-specific gap penalties and weight matrix choice. Nucleic Acids Res. 1994;22(22):4673-80 
29. Kumar S, Stecher G, Tamura K. MEGA7: molecular evolutionary genetics analysis version 7.0 for bigger datasets. Mol Biol Evol. 2016;33(7):1870-4.

30. Sun H, Pang B, Yan J, Wang T, Wang L, Chen C, Li Q, Ren Z. Comprehensive analysis of cucumber gibberellin oxidase family genes and functional characterization of CSGA200x1 in root development in Arabidopsis. Int J Mol Sci. 2018;19(10):3135.

31. Wilkins MR, Gasteiger E, Bairoch A, Sanchez JC, Williams KL, Appel RD, Hochstrasser DF. Protein identification and analysis tools in the ExPASy server. Methods Mol Biol. 1999;112(112):531-52.

32. Bailey TL, Nadya W, Chris M, Li WW. MEME: discovering and analyzing DNA and protein sequence motifs. Nucleic Acids Re. 2006;34:369-73.

33. Magali L, Patrice D, Gert T, Kathleen M, Yves M, Yves VDP, Pierre R, Stephane R. PlantCARE, a database of plant cis-acting regulatory elements and a portal to tools for in silico analysis of promoter sequences. Nucleic Acids Res. 2002:30(1):325-7.

34. Voorrips RE. MapChart: software for the graphical presentation of linkage maps and QTLs. J Hered. 2002;93(1):77-8.

35. Wang SS, Sun C, Liu ZZ, Shi QH, Yao YX, You CX, Hao YJ. Ectopic expression of the apple mhgai2 gene brings about GA-insensitive phenotypes in tomatoes. Acta Physiol Plant. 2012;34(6):2369-77.

36. Hichri I, Muhovski Y, Zizkova E, Dobrev PI, Franco-Zorrilla JM, Solano R, Lopez-Vidriero I, Motyka V, Lutts S. The Solanum lycopersicum zinc Finger2 cysteine-2/histidine-2 repressor-like transcription factor regulates development and tolerance to salinity in tomato and Arabidopsis. Plant Physiol. 2014;164(4):1967-90.

37. Liuhua Y, Qingzhe Z, Jianing W, Shuyu L, Bao W, Tingting H, Minmin D, Jiaqiang S, Le K, Chang-Bao L. Role of tomato lipoxygenase D in woundinduced jasmonate biosynthesis and plant immunity to insect herbivores. PLoS Genet. 2013;9(12):e1003964

38. Zhang H, Hu Z, Lei C, Zheng C, Wang J, Shao S, Li X, Xia X, Cai X, Zhou J. A plant phytosulfokine peptide initiates auxin-dependent immunity through cytosolic $\mathrm{Ca}^{2+}$ signaling in tomato. Plant Cell. 2018;30(3):652-67.

39. Zhang Y, Li D, Zhang H, Hong Y, Huang L, Liu S, Li X, Ouyang Z, Song F. Tomato histone H2B monoubiquitination enzymes SIHUB1 and SIHUB2 contribute to disease resistance against Botrytis cinerea through modulating the balance between SA- and JA/ET-mediated signaling pathways. BMC Plant Biol. 2015:15(1):1-20.

40. Xu S, Liao CJ. Tomato PEPR1 ORTHOLOG RECEPTOR-LIKE KINASE1 regulates responses to systemin, necrotrophic fungi, and insect herbivory. Plant Cell. 2018;30(9):2214-29.

41. Livak KJ, Schmittgen TD. Analysis of relative gene expression data using real-time quantitative PCR and the $2^{-\Delta \Delta C T}$ method. Methods. 2001;25:402-8.

42. Fillatti JJ, Kiser J, Rose R, Comai L. Efficient transfer of a glyphosate tolerance gene into tomato using a binary Agrobacterium Tumefaciens vector. Nat Biotechnol. 1987;5(7):726-30.

\section{Publisher's Note}

Springer Nature remains neutral with regard to jurisdictional claims in published maps and institutional affiliations.

Ready to submit your research? Choose BMC and benefit from:

- fast, convenient online submission

- thorough peer review by experienced researchers in your field

- rapid publication on acceptance

- support for research data, including large and complex data types

- gold Open Access which fosters wider collaboration and increased citations

- maximum visibility for your research: over $100 \mathrm{M}$ website views per year

At $\mathrm{BMC}$, research is always in progress.

Learn more biomedcentral.com/submissions 